\title{
COMPRESSION TEST OF THE MANDIBULAR CORTICAL BONE: A CADAVER STUDY ASSESSING FORMALIN TREATED \\ OR MACERATED BONE SPECIMENS
}

\author{
János Simonovics ${ }^{1}$, Péter Bujtár ${ }^{2}$, Károly Váradi ${ }^{1}$, Attila Szúcs ${ }^{3}$, Zsolt Fejér ${ }^{4}$ \\ ${ }^{1}$ Budapest University of Technology and Economics, Department of Machine and Product Design \\ ${ }^{2}$ University Hospitals of Oulu, Department of Oral and Maxillofacial Surgery \\ ${ }^{3}$ Semmelweis University, Department of Oro-Maxillofacial Surgery and Stomatology \\ ${ }^{4}$ Semmelweis University, Department of Human Morphology and Developmental Biology \\ janos@simonovics.hu
}

\begin{abstract}
Nowadays the examination of implants from engineering point of view is becoming more and more supported in the medical field. Instead of the more problematic cadaver examination, the computer based simulations are growing more popular. The examinations must be validated to be able to stay with the realistic situation.

Using these engineering methods it became possible to do measurements on the reconstructions of a human resectioned mandible. The creation of the bone models is one of the most varying fields in the biomechanical simulation. In the literature a wide range of Young modulus for the bone cortical layers can be found and these values are actively used even in different further finite element simulation researches. However the proper modeling of the cortical layer thickness and correctly calibrated mechanical parameter settings bare key importance regarding the stability and connection of the implants and the fixing screws.

The values of the Young modulus are depending on many factors, for example gender or even the age, not even mentioning further different influencing factors.

In this study destructive biomechanical examinations were carried out on bones received from dead people. The samplings of the bone were conducted from the area of the screwing used in the mandible reconstruction. Considering the results it can be concluded that the values in the literature are often not as accurate. Regarding orthotropic values of the mandible, so far has only been a small segment of information was covered.

The measured values are lower than the ones in the literature. In case of the highest value it is still 3.5 GPa Young modulus in the axial direction. The results can be used in order to create a more reliable model combined with a more realistic Young modulus for the finite element simulation of the bone models. On these models the different scenarios of the resection cases can be estimated more precisely with examinations.
\end{abstract}

Keywords: mandible, compression, Young modulus, bone

\section{Introduction}

In case of cancer, the more important factor is prevention. Narrowing it to the oral cavity cancers, most of them can be discovered with physical examination hence can be screened easily. The earlier the realization, the more lives can be saved. In Hungary every year approximately 1700 people die out of the 3000 discovered cases in the field of oral cavity can- 
cer. In light of these numbers we are on top of the list within Europe. In both female and male cases Hungary takes the number one spot considering the death toll from oral cavity cancers. ${ }^{1}$ Despite the easy detection of the problem most of the patients are too late, in which case a radical intervention cannot be avoided to save their lives, this way also reducing the chances of full recovery. The treatment of oral cavity cancers always depend on the location where it developed within the mouth, what is the extent of the development and how is the condition of the patient. When the jaw (mandible) is affected, the chances are very high that a surgical intervention is needed, during which the affected bone section will be cut out (resection).

During the resection the surgeon removes the bone allocation affected by the cancer. The resection can be partial or full depending on the extent of the bone. The effect on the mandible can be partial or full loss of function, further more often the mechanical load carrying role weakens or completely disappears.

The resection is unique in every case. It depends on the patient and the situation; therefore we cannot really distinguish between different scenarios from the surgeon point of view. However in most of the cases it can be divided into zones (Figure 1).

In the clinical practice there are two methods existing, which are highly dependent on the extent of the resection, furthermore on the structural quality of the remaining bones in the vicinity of the environment of the resection. One of these is the bone graft method. This can be used in case the bones are in good condition around the area of the resection. The fixation of the graft is realized with a plating technology.

In case of bones in worse condition or the extent of the resection is too big, only the plating technology can be used. There is a great variety of these plates exist. The thickness and length of these plates are selected by the surgeon based on the clinical case and needs of the patient. The length of the plate is selected considering the proper fixation with screws based on the extent of the resection. The plates are deformed based on the geometry of the bone in each case, therefore providing a unique shape for each patient. Each side of the resection usually 3-4 screws are used to fix the plates.

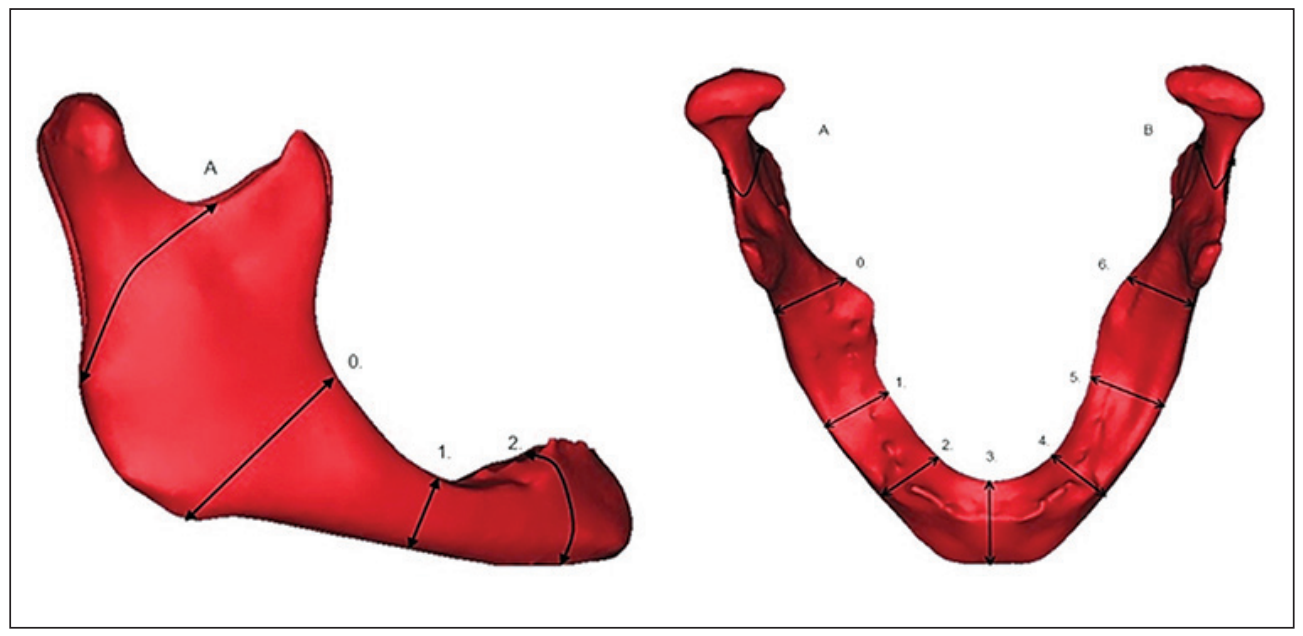

Figure 1. Important resection areas 
The screws can be divided into categories; monocortical and bicortical depending on the location and the size of the mandible section. The bicortical screw of course goes through the bone in such a depth that the connection in the counter cortical (hard) layer is big enough. In some cases it completely goes through, therefore the length of these screws is varying. Further we can distinguish among the new methods the so called Locking and Non-Locking systems. In case of the Locking system the screw head and the screw in the plate as well is created with sunken (low) threads, hence providing the secure fixation. However in the Non-Locking system only the plate holes are created with sunken (drawn) method therefore providing the appropriate connection.

The implants used in oral surgery are most of the cases made of an alloy of titanium (TiCP - titanium commercial pure, Ti6Al7Nb, etc.). The value of Young modulus is between 90$100 \mathrm{GPa}$ and the 0.3 Poisson coefficient.

\section{Methods}

The environment of the fixing screws for the implants is highly important considering the bones. The finite element simulations are becoming more and more popular in the biomechanical field compared to the often problematic cadaver examinations. In the models used for the simulations and also present in the literature are rarely or not at all considered the thickness of cortical allocation and the real mechanical properties. However these properties of the bone allocations can be shifted in a wide range and one by one can be very different. Examinations carried out with these models do not provide a realistic picture of the situation regarding the real relationship between the plates and screws.

For the examination the samples were selected from the area affected by the implant screws

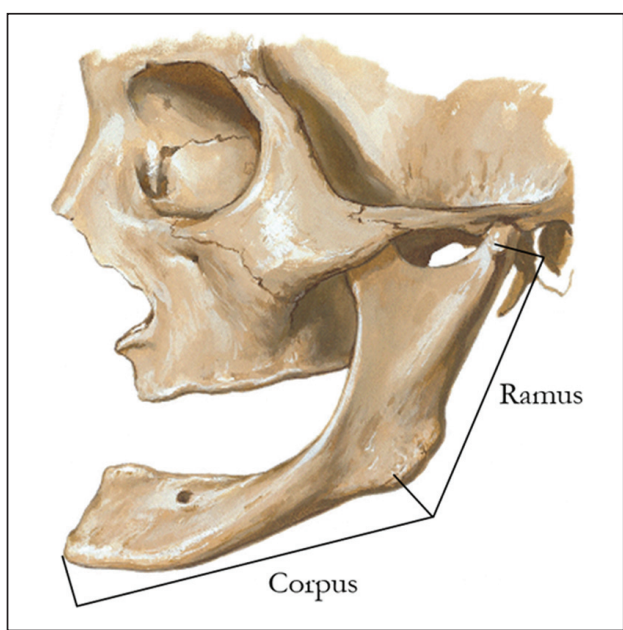

Figure 2. Toothless mandible of aged person

during the reconstruction. The samples were taken out of dead human formalin fixed and macerated mandibles. These samples were examined using a mechanical destructive method.

These screw locations in the clinical practice were also affected the corpus and ramus (Figure 2).

The purpose of the formalin fixation is to stop the decay of the enzymes. The water based formaldehyde is one of the most popular fixation methods. During the fixation the fixing solution/material penetrates the deeper regions of the tissues; therefore during the process the damage of the tissue structure is almost unavoidable. During the preparation using the macerated method while applying the warm water the soft regions are decomposed, the bone is steamed and whitened. The osteocollagen systems and salt of the bone stays intact during the preparation, therefore the mechanical properties do not change significantly. ${ }^{2}$

The sampling took place at the Semmelweis University of Human Morphology and Development Biology Department. The use of bone samples were approved by Committee of Sci- 


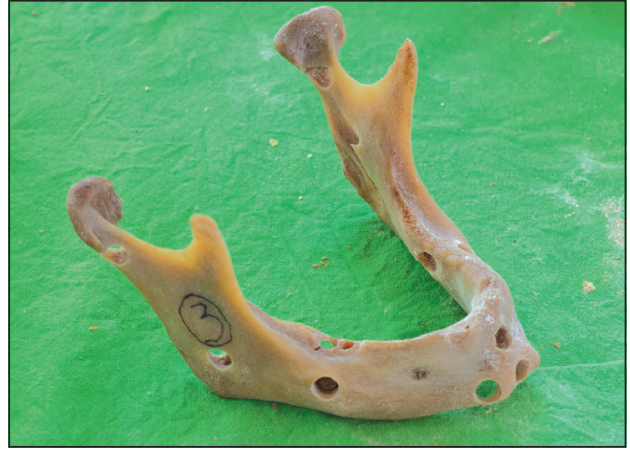

Figure 3. Sampling from macerated mandible

entific and Research Ethics. The heads kept in formalin from which the samples were taken are stored more than 2 years in formalin. The damage of the tissues was so high in some areas, where during the sanding process the samples basically dissolved by the high amount of load.

For the samples $6 \mathrm{~mm}$ inner diameter and $10 \mathrm{~mm}$ height bone segment was used, which was constantly cooled during the process. All together 96 helical samples was created from 3 formalin fixated and 6 macerated toothless aged human (male and female) mandibles (Figures 3-4).

The mandible is considered to be a complex bone because of its shape. The orthotropic structure is constantly changes with the geometry. ${ }^{3}$

The mechanical parameters from the cheek side and also from the oral cavity side can be entirely different, therefore when creating the samples the different orientations of the bones were marked. After the samples were created the spongiosa allocations were removed, than a special sending process was applied. As a result the samples took the shape of a square geometry with $4 \mathrm{~mm} \times 4 \mathrm{~mm}$ cortical thickness. After the structural storage in order to carry out the finite element simulations CBCT/CT scanning was performed on the samples with the help of a unique validation scheme. After

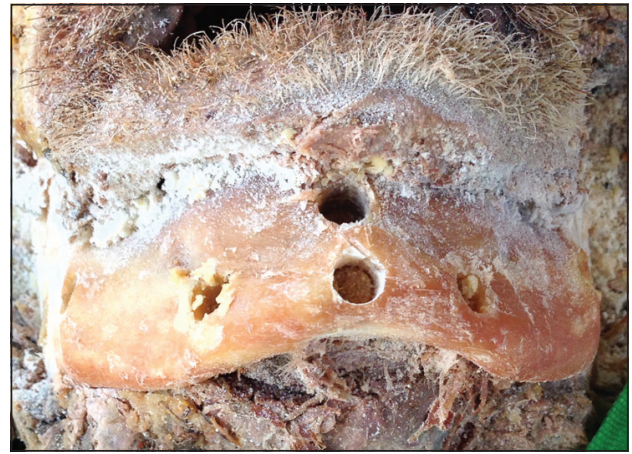

Figure 4. Sampling from formalin fixed mandible

the scanning randomly (disregard of sample location and order) compression examinations (pressing machine) were carried out at the Budapest University of Technology and Economics in the Cooperation Research Center for Biomechanics. During the examination the samples were divided into 3 sections and the orthotropic properties were observed focusing on the more precise determination of the Young modulus. Axial, radial and tangential orientations were examined based on the original position of the bone. As the expectation of the highest elasticity modulus was in the axial orientation, therefore the number of sampling was increased regarding the important macerated samples. As a result of the sanding process, many samples were rendered useless in the measurement aspect.

There were 80 from formalin fixed and macerated mandible samples which could be measured correctly, already excluding the ones that could not be worked with. The mixture of measurement orientations were the following (Table 1):

\begin{tabular}{|l|c|c|c|c|}
\hline $\begin{array}{l}\text { Measured samples } \\
\text { that can be evaluated }\end{array}$ & Axial & Radial & Tangential & Sum \\
\hline $\begin{array}{l}\text { Formalin fixed } \\
\text { mandible samples }\end{array}$ & 12 & 9 & 11 & 32 \\
\hline Macerated samples & 26 & 10 & 12 & 48 \\
\hline
\end{tabular}

Table 1. Examined samples 
Before the compression examination the geometrical measurement of each sample was done and documented. Force-displacement curve graphs were created from the results in

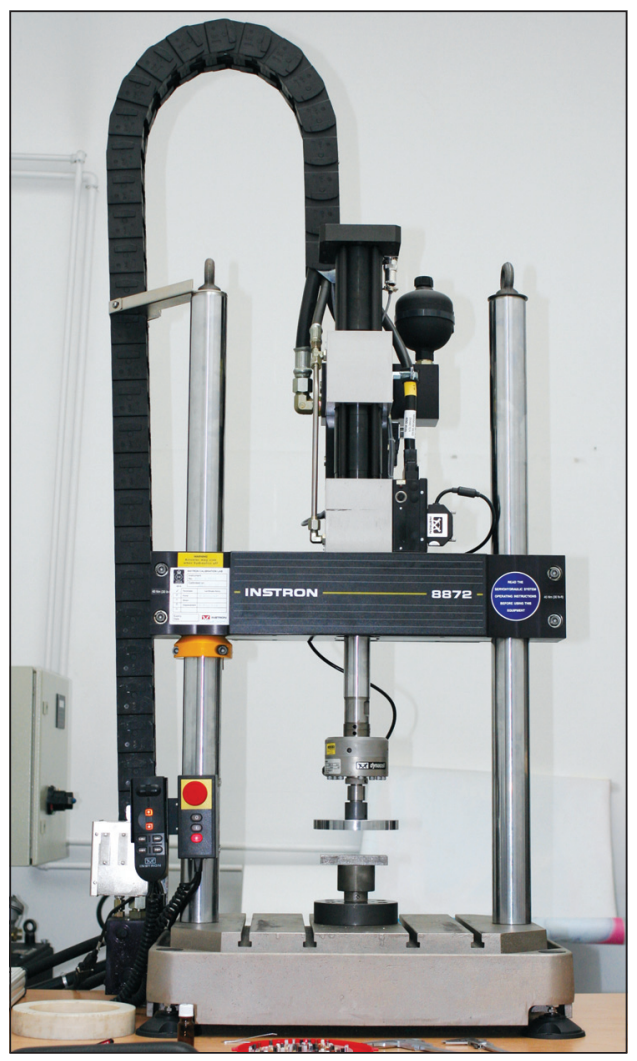

Figure 5. Compression test and a measured sample

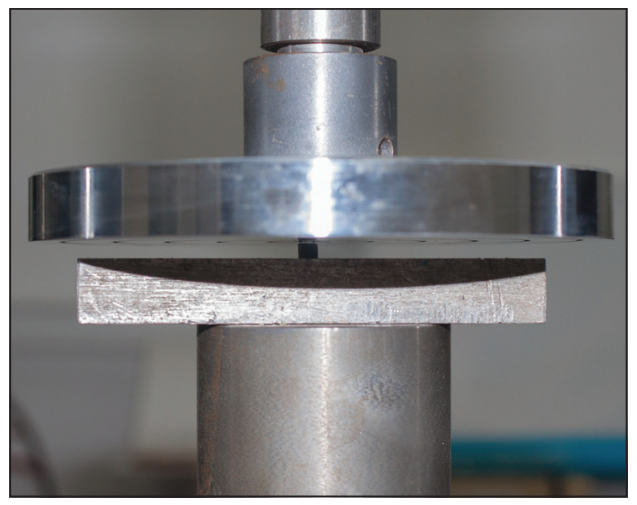

Figure 6. A measured bone sample light of the Young modulus consideration. The measurements were conducted within normal circumstances. The examination was carried out with a $25 \mathrm{kN}$ limited force measuring cell on an Instron 8872 machine (Figures 5-6). The load velocity was defined to be 0.5 , the measurements were conducted until the actual sample destruction based on the forcedisplacement curve.

The samples inside proper storage containers were sent back to the Semmelweis University Human Morphology and Development Biology Department, where they were finally destroyed.

\section{Results}

During the compression examination the force-displacement variables of the bone were registered (Figure 7). The data from the linear sections of the curve and the previously documented size parameters from the bone samples were used for the determination of the Young modulus.

The compression examinations on the bones found in the literature bear a high spread regarding the result data. This can be connected with the unique properties (gender, age and other factors) as well as the storage

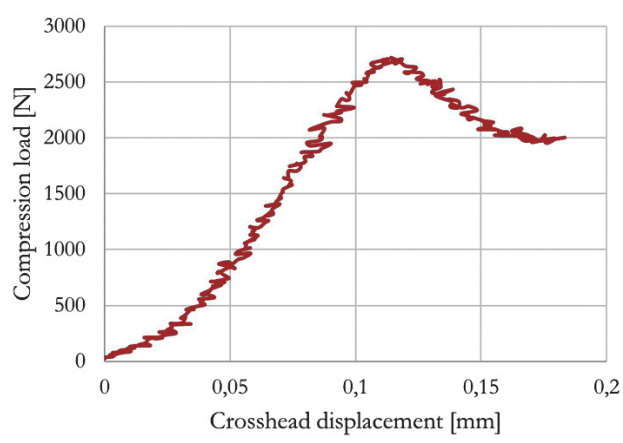

Figure 7. Load-displacement diagram of the $9^{\text {th }}$ macerated bone sample - axial compression 
of the bones. ${ }^{4}$ There is not much information available for the mandible mainly focusing on the orthotropic properties within the cortical allocations during the compression examination. Isotropic data can be found between 1.5$15 \mathrm{GPa}^{5}$ These values are below the average which can be found in the literature. In the technical literature refers to the average value of 14-23 GPa Young modulus, which is actively used for simulation purposes. ${ }^{6}$ On the other hand it is an overstatement to even use this for a mechanical simulation for example in a case of an aging human.
The current study shows that the values from the compression examination are below compared to the literature in both the formalin fixed and macerated bone cases. Hence the consideration of these values can be crucially important in order to create a more realistic model for example in case of a mandible reconstruction of an elderly human. Furthermore it is important from the mechanical point of view to divide properly the bone allocations and to define the material properties.?
Young modulus in axial direction in case of formalin fixed bone

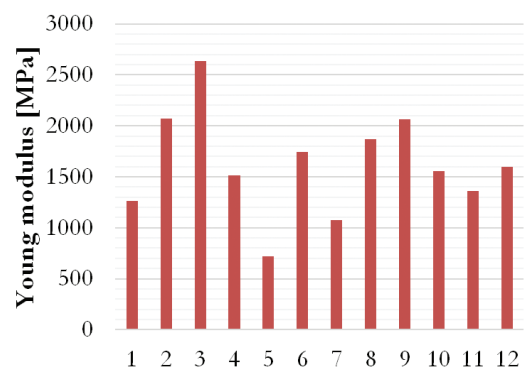

Measured samples in axial direction
Young modulus in axial direction in case of macerated bone

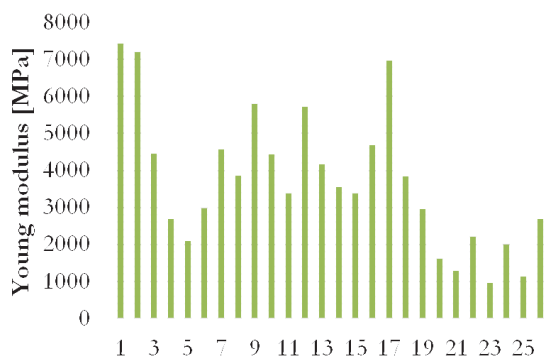

Measured samples in axial direction

Figure 8. Young modulus in axial direction

\section{Young modulus in radial direction in case of formalin fixed bone}

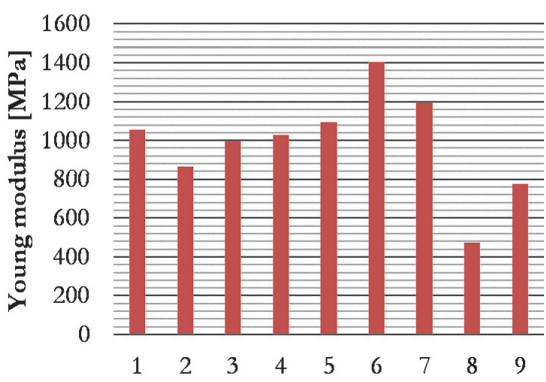

Measured samples in radial direction

\section{Young modulus in radial direction in} case of macerated bone

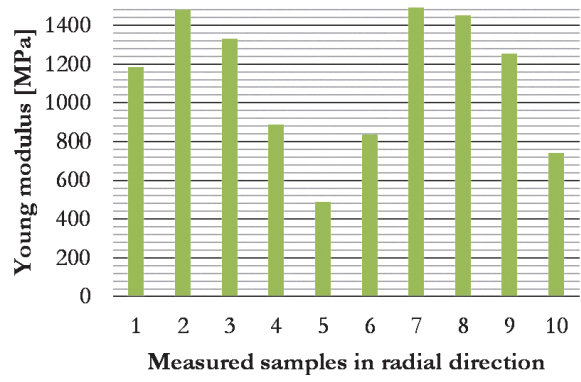

Figure 9. Young modulus in radial direction 


\section{Young modulus in tangential direction in case of formalin fixed bone}

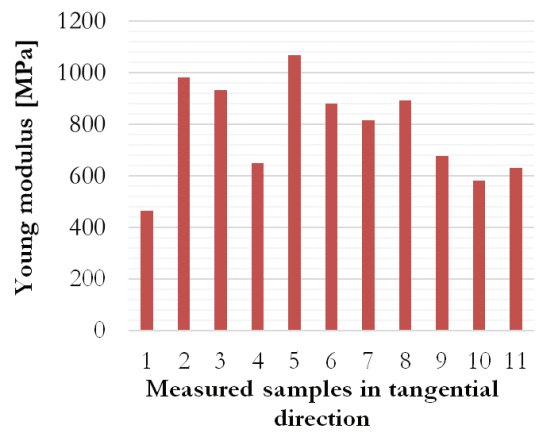

\section{Young modulus in tangential direction in case of macerated bone}

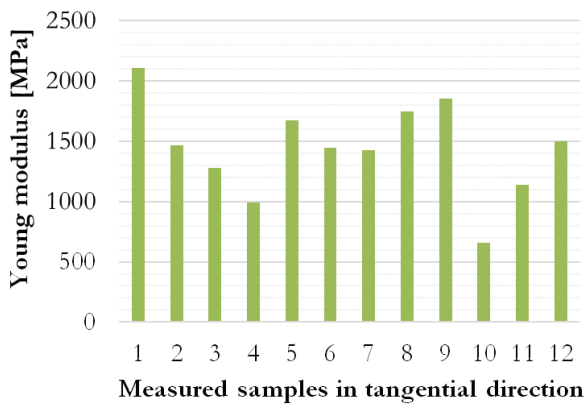

Figure 10. Young modulus in tangential direction

The measured values can be seen on the next diagram and for the sake of better understanding the different orientations and bone types were shown separately (Figures $8-10$ ).

The difference between the Young modulus, the average values and the spread in the values of different orientations shown in a box-plot diagram can be seen quite well in both cases of the formalin fixed and macerated samples (Figure 11). On the diagram considering the
$50 \%$ median, the lower and upper percentile of 25 and $75 \%$ was defined (quartile). This way making more strict the generally used normal domains of $2.5-97.5 \%$ and the $5-95 \%$. It is transparent that the mechanical properties of the formalin fixed mandible samples were changed during the fixing. Substantial tissue damage could have occurred in case of the samples due to the storage for possibly more than 2 years. The axial orientation produced more favorable values in both sample cases

Young modulus in different directions in case of formalin fixed bone

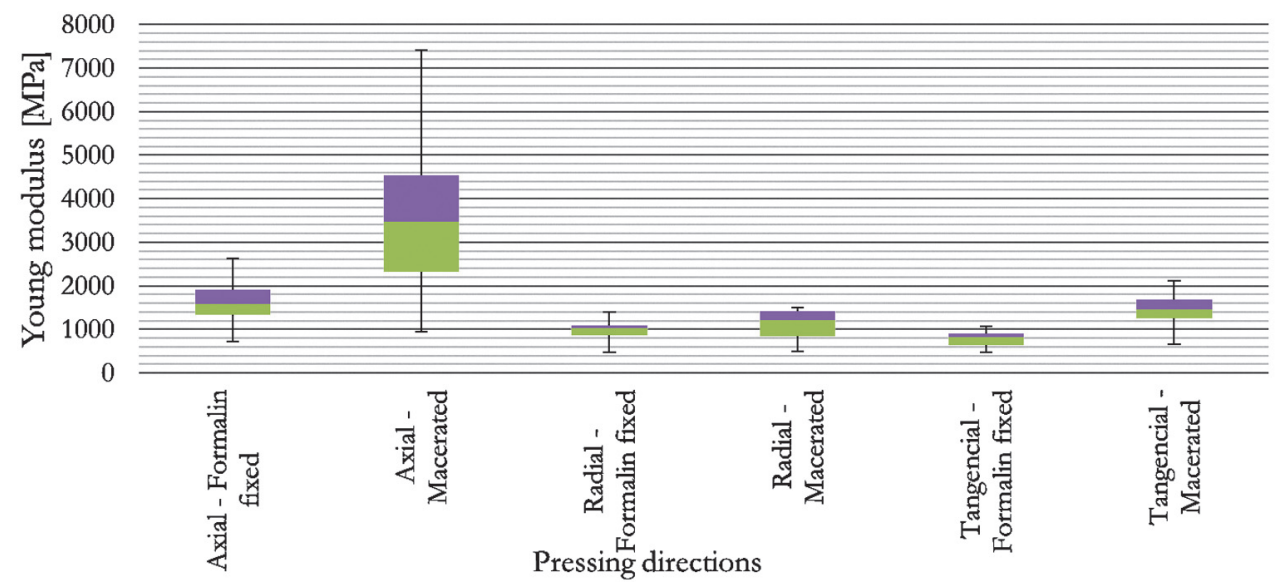

Figure 11. Young modulus in different directions in case of formalin fixed bone 
than the radial and tangential orientation, conforming to the different bone types found in the literature. ${ }^{8,9}$ The tangential orientation in some measurements of the macerated samples showed better properties than the radial orientation, focusing on the Young modulus. In case of the formalin fixed samples the situation is reversed. The radial orientation compression examination shows better values in the Young modulus.

\section{Summary}

Medical treatment of jaw cancer becomes more and more important as the number of diagnosed patients has risen significantly. It is inevitable to understand the correct properties of the mandible in order to provide an adequate reconstruction. Cadaver examinations are available to determine the mechanical parameters.
During this study a compression examination was carried out in orthotropic orientations on toothless mandibles from dead humans both on formalin fixed and macerated samples. The determined Young modulus values registered after the examinations are greatly differing from the values that can be found in the general literature. The results from the samples with significantly different mechanical properties could have key importance in further biomechanical examinations. In the field of model creation the results show that the uniquely changing mechanical properties cannot be excluded in order to create a more realistic model. The collected results can be well used in the finite element analysis method that is becoming more and more popular. These examinations can provide better estimations in the other areas of oral surgery regarding the possible loads. Considering the possibility of improvement, the probability of more stability and faster recovery can be provided for the patients.

\section{REFERENCES}

1. Levi F, Lucchini F, Negri E, Boyle P, La Vecchia C. Cancer mortality in Europe, 1995-1999, and an overview of trends since 1960. Int J Cancer 2004 Jun 10;110(2):155-69.

2. Szentágothai J, Réthelyi M. Funkcionális anatómia. Budapest: Medicina Könyvkiadó Rt.; 2002.

3. Nomura T, Gold E, Powers MP, Shingaki S, Katz $J L$. Micromechanics/structure elationships in the human mandible. Dent Mater 2003;19(3):16773.

4. Thomsen JS, Niklassen AS, Ebbesen EN, Brüel A. Age-related changes of vertical and horizontal lumbar vertebral trabecular 3D bone microstructure is different in women and men. Bone 2013 Nov;57(1):47-55.

5. Nakajima K, Kondoh J, Fujiwara M. An experimental study on the dynamic traits of dehydrated mandibles in relation to Yang's modulus and Poisson's ratio of compact bone. Shika Gakuho Dent Sci Rep 1984 Dec;84(12):1951-61.

6. Kimura A, Nagasao T, Kaneko T, Tamaki T, Miyamoto J, Nakajima T. Adaquate fixation of plates for stability during mandibular reconstruction. J Cranio-Maxillofac Surg 2006 Jun;34(4):193-200.

7. Simonovics J, Bujtár P, Váradi K. Effect of preloading on lower jaw implant. Biomechanica Hungarica 2013;6(1)21-28.

\section{Lawrence Katz J, Yoon HS, Lipson S, Maharidge} $R$, Meunier A, Christel P. The effects of remodeling on the elastic properties of bone. Calcif Tissue Int $1984 \mathrm{Mar} ; 36(\mathrm{~S} 1): S 31-\mathrm{S} 36$.

9. Meunier A. Scanning acoustic microscope studies of the elastic properties of osteons and osteon lamellae. J Biomech Eng 2008 Mar 17;115(4B):543. 
We would like to give my sincere gratitude for the Budapest University of Technology and Economics in the Cooperation Research Center for Biomechanics for the possibility to use the measurement equipments. I would also like to thank Péter Mészáros for his unwavering help and support.

\section{János Simonovics}

Budapest University of Technology and Economics, Department of Machine and Product Design H-1111 Budapest, Bertalan Lajos u. 1-3.

Tel.: (+36) 303736654 